\title{
Chickadee Songs Provide Hidden Clues to Singers' Locations
}

\author{
Eduardo Mercado III ${ }^{1 *}$, Matthew G. Wisniewski ${ }^{1}$, Brittany McIntosh ${ }^{1}$, Lauren M. Guillette ${ }^{2}$, \\ Allison H. Hahn' ${ }^{2}$, and Christopher B. Sturdy ${ }^{2}$
}

${ }^{1}$ University at Buffalo, The State University of New York, New York, USA

${ }^{2}$ University of Alberta, Edmonton, Alberta, Canada

*Corresponding author (Email: emiii @buffalo.edu)

Citation - Mercado, E. III, Wisniewski, M. G., McIntosh, B., Guillette, L. M., Hahn, A. H., \& Sturdy, C. B. (2017). Chickadee songs provide hidden clues to singers' locations. Animal Behavior and Cognition, 4(3), 301-313. https://doi.org/10.26451/abc.04.03.08.2017

\begin{abstract}
Coordination of actions requires that organisms actively monitor the movements of others. The current study examined acoustic cues within the fee-bee song of chickadees that may provide listening conspecifics with information about the movements of singers. The difference between direct and reverberant acoustic energy present during the second note of the fee-bee song provided clear indications of how far the song had traveled. Preliminary analyses suggest that this distance cue may be robust to variations in the spectra and amplitude of song components, and that the acoustic features of the fee-bee song may facilitate simultaneous comparisons of reverberating fees with directly received bees by listening birds. Comparing coincident reverberation with directly received sounds may be a previously unsuspected way that animals living in reverberant environments can monitor the movements and interactions of conspecifics.
\end{abstract}

Keywords - Ranging, Distance, Spatial hearing, Vocalizations, Singing, Black-capped chickadee, Songbird

To interact successfully, an organism must assess the actions of other individuals and respond accordingly. Individuals occasionally can use visual information about the movements of others to accomplish this, but in some situations, they must rely on information from other modalities. For instance, whales and dolphins cooperatively forage in situations where they are unlikely to be able to see conspecifics (Connor, 2000), and birds defend territories in wooded environments where familiar neighbors and unfamiliar intruders are only intermittently visible (e.g., Fitzsimmons, Foote, Ratcliffe, \& Mennill, 2008). In these contexts, sounds can provide important clues about the actions of others, including where they are going and what they are doing.

How animals use sounds to monitor and manipulate the actions of others has been studied most extensively in territorial songbirds. For a bird to be able to exclude other birds from a specific area, it must be able to judge when those birds are intruding - this often requires that the territory defender estimate other birds' distances based on received sounds. Birds use several acoustic cues to do this, including variations in overall amplitude, differential spectral attenuation, and signal distortions associated with reverberation (reviewed by Naguib \& Wiley, 2001). Some acoustic features of songs, such as rapid trills, degrade rapidly as they propagate, suggesting that these features may function, at least in part, to provide information about a vocalizing bird's distance from any listening birds (Naguib, 2003; Wiley \& Richards, 1978). Judging the distance to a vocalizing individual is complicated by the fact that 
not all individuals produce sounds at the same intensity or with identical spectrotemporal features; absolute values of received features thus cannot unambiguously reveal a singing bird's location.

Most past studies of auditory distance estimation by birds (referred to as ranging) have emphasized the need for listening birds to compare received sounds with properties of the original sound features produced at the source (e.g., Fotheringham \& Ratcliffe, 1995; Nelson \& Stoddard, 1998; Wiley, 1998). Because a listener cannot simultaneously perceive a sound at its source and at a distance, such comparisons require that listeners compare heard sounds to internal representations of undistorted songs or calls (Morton, 1986, 2012). Knowledge of source features is not always a prerequisite for assessing auditory distance, however. For instance, echoes generated by environmental reflections can provide information about source distance that does not depend on knowledge of source features (Nelson \& Stoddard, 1998). Similarly, by sequentially comparing directly received sounds to the reverberation generated by those sounds, listeners can estimate the range of a sound source without knowing the intensity of the source (Larsen, Iyer, Lansing, \& Feng, 2008; Zahorik, 2002). Past assessments of the role reverberation plays in auditory distance estimation by birds have typically focused on how reverberation distorts song features (Dabelsteen, Larsen, \& Pedersen, 1993; Fotheringham \& Ratcliffe, 1995; Holland, Dabelsteen, Pedersen, \& Paris, 2001; Nelson \& Stoddard, 1998; Wiley \& Richards, 1978). However, reverberation can also enhance long-distance transmission if the energy within sounds is focused within a narrow frequency band (Slabbekoorn, Ellers, \& Smith, 2002).

Black-capped chickadees (Poecile atricapillus) provide a model system for exploring how singing birds may use sound to track the movements of conspecifics. Each spring, chickadees form neighborhoods of breeding pairs, within which they actively defend wooded areas covering 1-13 acres (Mennill \& Otter, 2007; Stefanski, 1967). Countersinging interactions appear to be an important component of territory defense by chickadees, and most such interactions involve singers either moving toward or away from each other (Dixon \& Stefanski, 1970; Fitzsimmons et al., 2008). Songs appear to be particularly important for long-range interactions, because chickadees in territorial disputes often switch to producing calls when they are in visual contact (Dixon \& Stefanski, 1970; Ficken, 1981). The songs produced by black-capped chickadees typically conform to a single type (referred to as a "fee-bee" song) consisting of two tonal notes. Based on a combination of playback studies and acoustic experiments, Fotheringham and Ratcliffe (1995) suggested that the complexity of chickadee habitats precluded ranging based on song degradation cues (see, however, Lohr, 2008), raising the question of how singing chickadees might use songs to coordinate their movements.

The goal of the present study was to evaluate whether chickadees might be able to use cues within received songs other than overall amplitude, frequency range, or degradation-related cues to judge a singer's distance. We predicted that reverberant tails from fee notes that coincided with bee notes could provide useful information about how far a song had travelled. Furthermore, we hypothesized that the acoustic structure of the fee-bee song facilitates the kinds of simultaneous, across-frequency comparisons that a listening chickadee would need to make in order to range singers using such cues.

\section{Method}

\section{Subjects}

Fee-bee songs were recorded from ten captive, adult black-capped chickadees, five male and five female. Each bird was placed within a cage $(30 \times 40 \times 40 \mathrm{~cm})$ that was housed within a sound-attenuating chamber (Industrial Acoustics Co. Inc.; inner dimensions $58 \times 168 \times 83 \mathrm{~cm}$ ). Songs were recorded (mono, 16 bit, $44.1 \mathrm{kHz}$ ) using an AKG C 1000s condenser microphone (AKG Acoustics GMbH) located $40 \mathrm{~cm}$ above the top of the cage connected to a Marantz PMD 670 digital recorder (Marantz America Inc.). 


\section{Procedure}

A sound transmission experiment was conducted in an open field during the spring season in Buffalo, NY. Measurements were made at night in order to minimize interference from local singing birds. One song from each bird was broadcast and recorded at multiple distances. A Roland JC-120 amplifier (Roland Co.) was used to broadcast the songs through a 12" Roland speaker at a height of 1.5 $\mathrm{m}$. Levels of fee-bee song playbacks were measured at $80 \pm 3 \mathrm{~dB}$ SPL, $1 \mathrm{~m}$ away from the speaker with a RadioShack 33-4050 analog sound level meter (RadioShack Co.), so that playback levels were comparable to those naturally produced by chickadees (Christie, Mennill, \& Ratcliffe, 2004a, b; Nowicki, 1983). A Zoom (Zoom Co.) H4 digital recorder (48 kHz sampling rate) equipped with two unidirectional condenser microphones (frequency response range $=20 \mathrm{~Hz}$ to $20 \mathrm{kHz}$ ) was mounted on a microphone stand at a height of $1.5 \mathrm{~m}$. Broadcasts were recorded at distances of 2, 10, 20, 40, 60, and $80 \mathrm{~m}$ from the speaker.

\section{Data Analyses}

Recordings were analyzed using Raven Pro 1.4 for Mac OS X by manually selecting individual fee or bee notes and automatically measuring peak frequency and peak power from each note (Figure 1). Additionally, the bee note was analyzed a second time with all spectral energy other than energy at the peak frequency of the initial fee note bandpass-filtered, to determine the peak power for reverberation from the fee note present during reception of the bee note. Measures of differences between peak power at the peak frequency in the bee note and peak power at the peak reverberated frequency from the fee note were calculated and analyzed as a function of recording distance.

The features of recorded songs at the source were also visualized using an auditory spectrogram in which filter spacing and bandwidths matched auditory filter bandwidths measured electrophysiologically from Carolina chickadees (Henry \& Lucas, 2010).

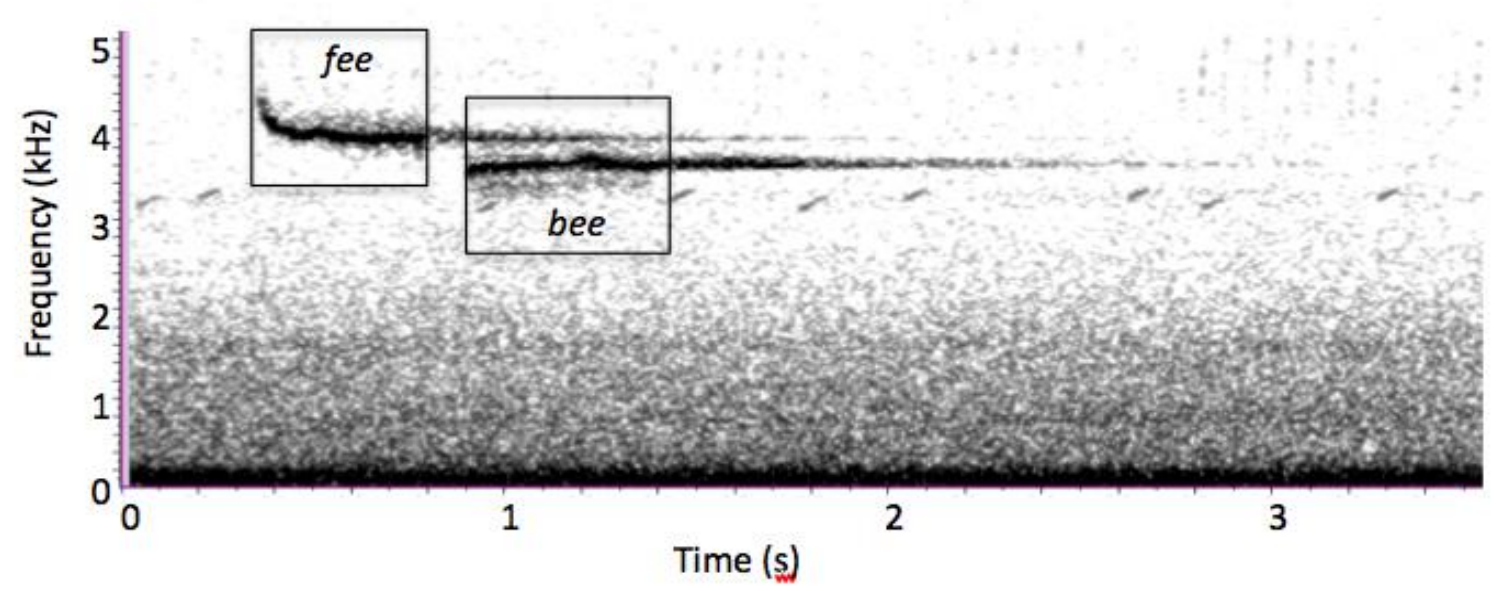

Figure 1. A fee-bee song recorded from a distance of $80 \mathrm{~m}$ in an open field. Note that the duration of reverberant tails for each note is comparable to the duration of the entire song. Boxes illustrate how individual notes were manually selected prior to automated measurement of peak frequencies and peak power (FFT $=2048,98 \%$ overlap; Hanning window).

\section{Results}

Analyses of the energetic features of fee-bee songs recorded at close ranges $(2 \mathrm{~m})$ showed that peak power was focused within a narrow band for each note for each singer ( fee note range $=3.2-3.9$ 
$\mathrm{kHz}$; bee note range $=2.9-3.6 \mathrm{kHz}$ ). The specific bands varied across singers, but the ratio of the two bands was comparable across individuals (mean ratio $=1.11, S D=0.04$; Weisman \& Ratcliffe, 2004, reported a mean ratio of 1.134 from a sample of 154 wild birds). Reverberant tails clearly revealed the narrow bands within which energy was focused for both notes (Figure 1). Tails were evident at all recorded distances between $10-80 \mathrm{~m}$ and extended well beyond the duration of each note. In particular, the reverberant tail from the initial fee note persisted beyond the duration of the following bee note, such that energy from both narrow bands was evident within spectral analyses of the bee note (Figure 2A). Peak power within the fee and bee notes decreased systematically with distance (Figure 2B). Transmission loss was comparable across songs from different birds, despite individual variation in the frequency bands where song notes were focused.

Peak power from reverberation of the fee note also decreased systematically with distance (Figure 2B), but transmission loss was less than for either directly received note. Consequently, the difference between the peak power of the directly received bee note and the peak power within the reverberant tail from the fee note was negatively correlated with distance from the source $(r=-0.86, p=0.028)$. Auditory spectrographic analyses of the fee-bee song (Figure 2C) revealed that the notes within the song were spaced the minimal amount necessary to activate two adjacent auditory filters.

\section{Discussion}

Results from the current transmission experiment indicate that reverberation may facilitate auditory distance estimation by enabling listening chickadees to compare long-lasting reverberant energy from the first note in a fee-bee song with simultaneously received acoustic energy from the second note. By detecting any changes in this distance cue that occur across consecutive songs, listeners can potentially monitor the movements of singers when they are beyond visual range.

\section{Cues to Singer Distance}

Researchers have identified several acoustic cues that birds use, or could use, to determine the distance to a singer (Table 1). Mammals make use of many of these same cues when judging auditory distance (Kolarik, Moore, Zahorik, Cirstea, \& Pardhan, 2016). Some cues provide information only about the relative distance to a source, whereas others can provide more specific information about the absolute distance (Naguib \& Wiley, 2001). In some cases, acoustic cues to distance may be useful only within a familiar environment (Nelson \& Stoddard, 1998). The three acoustic cues that have attracted the most scientific attention to date are variations in song amplitude, frequency content, and reverberation-related degradation (Fotheringham \& Ratcliffe, 1995; Phillmore, Sturdy, \& Weisman, 1998; Pohl, Klump, \& Langemann, 2015; Radziwon, Welch, Cone, \& Dent, 2011).

Songs become quieter as they travel farther. Birds clearly can make use of amplitude cues to judge singer distance in some circumstances (Nelson, 2000; Radziwon et al., 2011). Amplitude cues are less reliable than other distance cues because of amplitude variations caused by factors other than source distance, such as differences in source levels, atmospheric conditions, and height of the singer (Morton, 1982; Wiley \& Richards, 1978, 1982). Chickadees trained to discriminate songs recorded from different distances varied in their use of amplitude cues, with some individuals using them and others ignoring them (Phillmore et al., 1998).

Past considerations of spectral energy as a possible cue for assessing the distance to a singing bird note that higher frequencies often attenuate more rapidly than lower frequencies in bird habitats (Wiley \& Richards, 1978). Using spectral attenuation to judge auditory distance requires detailed knowledge of the frequency content of the song at the source. For chickadee song, researchers suggested that the initial frequency in the frequency-modulated (FM) down-sweep that starts each fee note (Figure 1) will degrade rapidly with distance such that the bandwidth of FM in a received fee note might provide a distance cue to listeners (Shackleton, Ratcliffe, \& Weary, 1992). However, in highly reverberant habitats, such as those frequented by black-capped chickadees (Smith, 1991), constructive and destructive interference can 
introduce fluctuations in frequency attenuation that confound interpretation of variations in the spectra of received songs. Using acoustic transmission studies, Fotheringham and Ratcliffe (1995) found that differential frequency attenuation in fee-bee songs did not reliably indicate source distance (however, see Christie et al., 2004a).

(a)
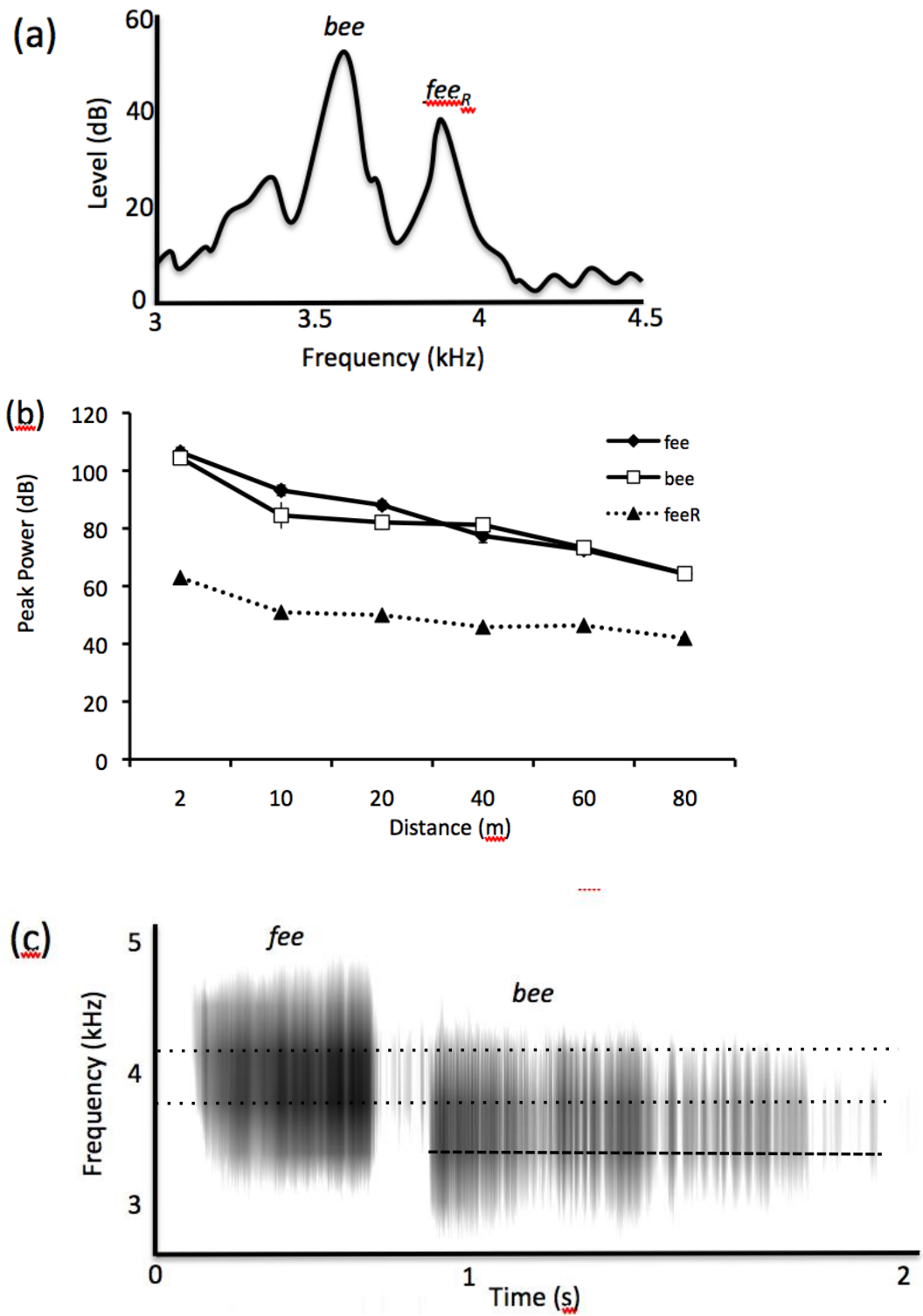

Figure 2. (A) Spectral peaks are evident both from the directly received bee note and from the reverberant tail of the preceding fee note $\left(f_{e} e_{R}\right)$ after a song has traveled $80 \mathrm{~m}(\mathrm{FFT}=2048$; 98\% overlap; $250 \mathrm{~ms}$ Hanning window). (B) Differences in power at the peak frequency between the bee note and the reverberated fee note decreased systematically with distance. Data points are means from ten songs; standard error bars for most points are not visible because of low variation across broadcasts. (C) An auditory spectrogram of a fee-bee song that corresponds to a chickadee's auditory filter bandwidth at $3.5 \mathrm{kHz}(\sim 600 \mathrm{~Hz}$; see Figure 1 for a more standard, high-resolution spectrogram) shows that the frequency spacing between the fee and bee notes corresponds closely to the spacing between two adjacent auditory filters (dashed and dotted lines delineate processing by adjacent filters; FFT $=115,98 \%$ overlap, Hanning window). 
Table 1

Potential Auditory Distance Cues within Chickadee Fee-Bee Songs

\begin{tabular}{ll}
\hline Distance Cue & Limitations \\
\hline Note amplitude & Varies with source amplitude and position \\
Fee frequency spectrum & Changes are small in chickadee habitats \\
Inter-note duration & Varies with source duration and habitat \\
General degradation & Changes are small in chickadee fee-bee song \\
Amplitude fluctuations & Highly variable across different habitats \\
Direct-to-reverberant energy ratio & Requires isolating signal from reverberation \\
Reverberation amplitude & Varies with source amplitude and position \\
Reverberation frequency spectrum & Changes are small in chickadee habitats \\
Environmental echoes & Cues are location-specific \\
Source elevation & Listener must know source height \\
Coincident reverberation & Relative amplitude of two notes must be consistent \\
\hline
\end{tabular}

Reverberation of a song during transmission can lead to a variety of different spectral and temporal cues that are indicative of source distance (Holland et al., 2001; Naguib, 2003; Richards \& Wiley, 1980). In chickadee songs, these cues may include the duration of individual notes, the duration of the interval between notes, degradation of the general frequency contours, and the presence or absence of breaks within individual notes (Fotheringham \& Ratcliffe, 1995; Lohr, 2008). As with the frequency content of songs, the value of each of these cues depends on a listener's knowledge of what song features were originally produced by the singer. The one attempt at varying reverberation-related cues during playbacks of songs in the presence of wild chickadees found that listeners showed comparable responses to both degraded and undegraded songs (Fotheringham \& Ratcliffe, 1995).

In the current study, broadcast fee-bee songs showed distance-related changes in the difference between the amplitude of the frequency with maximum amplitude from the second received note (the bee) and the amplitude of the frequency with maximum amplitude from the reverberant tail generated by the first note (the fee). Specifically, the difference between the two spectral peaks decreased as the distance from the source increased. Unlike all previously noted distance cues, estimating source distance from this difference cue (hereafter referred to as a coincident reverberation cue) does not require knowledge of the source level, frequency content, or frequency contour produced by the singer. In other words, if a listening chickadee used this cue to estimate a singer's distance, then the estimate should not change if the singer shifts its song notes to different frequencies or increases overall song amplitude, because the relative difference between spectral peaks within the received song would remain constant despite such changes. In the case of coincident reverberation cues, reverberation facilitates auditory distance estimates not because of song distortion, but because it preserves acoustic information generated by the initial fee note (see also Slabbekoorn et al., 2002).

\section{Perception of Changes in Singer Distance}

Coincident reverberation has yet to be directly manipulated in playback experiments, making it difficult to assess if and when listening chickadees might attend to this cue. To our knowledge, possible use of coincident reverberation cues in auditory distance judgments has never been investigated in any species. Humans have been tested on their ability to detect differences in the intensities of two simultaneously presented tones centered at different frequencies, however (Dai \& Green, 1992; Feth \& O'Malley, 1977; Versfeld \& Houtsma, 1995). Such distinctions can be viewed as a special case of profile analysis (Green, 1987), which is closely related to auditory discrimination of spectral shape (Bernstein \& Green, 1988; Bregman, Patel, \& Gentner, 2016; Langemann, Zokoll, \& Klump, 2005). Profile analysis involves detecting an absolute increment in the amplitude of one tonal signal among a background of 
other tonal signals. Spectral shape discrimination similarly involves distinguishing patterns in spectral levels; recognition of such differences is thought to contribute to timbre perception (Cynx, Williams, \& Nottebohm, 1990; Hoeschele, Cook, Guillette, Hahn, \& Sturdy, 2014; Lohr \& Dooling, 1998). In the case of coincident reverberation cues within received fee-bee songs, both directly received and reverberant spectral peaks decrease in amplitude with distance, but at different rates, so that the relative difference in amplitude between the two peaks gradually decreases with distance.

Recent experimental studies show that birds are more sensitive to similarities and differences in spectral shape than was previously assumed (Bregman et al., 2016; Hoeschele et al., 2014). Chickadees, in particular, appear to find relative differences in the amplitudes of frequencies produced within fee-bee songs to be highly salient (Hoeschele et al., 2014). These findings suggest that chickadees are likely to perceive variations in the relative intensities of the spectral peaks that are present during reception of a bee note. But, these findings do not address the resolution with which listening birds might discriminate or identify particular spectral shapes, which would determine the precision with which listeners could map received sounds to different source distances. Humans trained to discriminate between two-tone sounds were able to discriminate level differences as small as $0.2-0.5 \mathrm{~dB}$, even when the average intensity level of the tones was roved across $20 \mathrm{~dB}$ (Versfeld \& Houtsma, 1995). Humans are most sensitive to differences in the intensity of simultaneously presented sinusoidal tones when one tone is just outside the critical band of the other tone (Dai \& Green, 1992; Feth \& O'Malley, 1977).

Chickadees undoubtedly can recognize the acoustic features of fee-bee songs and detect differences in those songs (Hahn et al., 2016, 2017; Hoeschele et al., 2010; Phillmore, Sturdy, Turyk, \& Weisman, 2002; Phillmore et al., 1998). To make use of coincident reverberation cues in estimating the distance to a singer, a listening chickadee would need to be able to detect and discriminate relatively small differences in intensity levels across the two frequency bands associated with fee and bee notes. Interestingly, the peak frequencies of fee and bee notes fall within the region of greatest auditory sensitivity in chickadees (Wong \& Gall, 2015), and the spacing between these two peaks matches the width of auditory filters in a listening chickadee's peripheral auditory system (Figure 2C; Henry \& Lucas, 2010). Because singing chickadees maintain a relatively fixed frequency ratio between the two notes when they shift their songs up or down in frequency (Weisman \& Ratcliffe, 1989, 2004; Weisman, Ratcliffe, Johnsrude, \& Hurly, 1990), the relationship between peak frequency spacing and auditory filter bandwidth holds true for all fee-bee songs. This correspondence between peak frequency spacing and auditory resolution suggests that the acoustic structure of the fee-bee song may facilitate discrimination of coincident reverberation cues by listening birds.

The ability of chickadees to reliably estimate singer distance based on coincident reverberation cues does not depend on knowledge of source characteristics, but it does require that the singer produce notes at consistent amplitude levels. If there are unpredictable differences between the sound pressure levels of fee and bee notes across repetitions, then this will lead to changes in relative differences between the levels of spectral peaks that are unrelated to source distance. For example, if a singing chickadee gradually decreased the intensity of its fee note relative to its bee note across song repetitions, then this could lead to gradual changes in received coincident reverberation cues that would be comparable to those produced by a singer with a more consistent song that is moving toward the listener. Conversely, if a singer were to attenuate its bee note relative to its fee note (as singing chickadees often do when they produce a short break midway through their bee note), then this would generate coincident reverberation cues similar to those that would occur if the singer were farther from the listener. Intriguingly, recent acoustic analyses show that dominant singing chickadees reliably produce songs with comparable spectral energy in their fee and bee notes (Hoeschele et al., 2010), providing a stable baseline for cross-note comparisons. Experimental studies of timbre discrimination in zebra finches and budgerigars show that they can detect changes of $1-3 \mathrm{~dB}$ in harmonic levels, which is slightly better than performance by humans (Cynx et al., 1990; Lohr \& Dooling, 1998). Human sensitivities for detecting such changes in two non-harmonic tones predict that deviations in the range of $0.2-0.5 \mathrm{~dB}$ are potentially discriminable by birds and other vertebrates. Collectively, these features of fee-bee song production and perception 
strongly suggest that chickadees should be capable of judging differences in coincident reverberation cues with sufficient accuracy to provide useful information about a singer's location.

Use of coincident reverberation cues for estimating source distance is complicated by the fact that both transmission loss and reverberation vary across habitats (Huisman \& Attenborough, 1991; Richards \& Wiley, 1980; Yang, Kang, Cheal, Van Renterghem, \& Botteldooren, 2013), and as a function of source and receiver positions (Nelson \& Stoddard, 1998; Padgham, 2004), such that a received spectral shape might be associated with different absolute distances in different habitats. For example, spectral peaks present in the bee note might be equal at a distance of $80 \mathrm{~m}$ in one environment, but not in a different habitat. Many environmental variables affect how songs attenuate and reverberate during transmission (Richards \& Wiley, 1980; Wiley \& Richards, 1978, 1982). Because most of the conditions that affect propagation along a direct path will also affect transmission of reverberated echoes along indirect paths, habitat-dependent effects on attenuation will be correlated with habitat-dependent effects on reverberation. Nevertheless, a listening bird must either account for possible habitat-dependent effects on received signals, or learn through experience how changes in spectral shape relate to variations in singer distance within a particular habitat, to accurately map coincident reverberation cues onto different source distances. This constraint applies to all other known auditory distance cues as well, and so does not require listening birds to possess any processing capacities beyond those that they are currently assumed to have. Habitat-dependent propagation effects are less likely to affect a listening bird's ability to track a singer's movements, because if a singer is stationary, approaching, or leaving while singing multiple songs, then the difference between reverberation and a directly received note will remain stable, increase, or decrease accordingly.

\section{Relevance for Coordinating Actions}

Chickadees produce and use songs differently from many other songbirds (Dixon \& Stefanski, 1970; Ficken, 1981; Mennill \& Otter, 2007; Odum, 1941; Stefanski, 1967). Black-capped chickadees' entire song repertoire typically consists of a single two-note song (for exceptions, see Gammon \& Baker, 2004; Gammon, Baker, \& Tipton, 2005; Kroodsma et al., 1999). They use this song year-round, in a wide variety of behavioral contexts (Avey, Quince, \& Sturdy, 2008; Dixon \& Stefanski, 1970; Fitzsimmons et al., 2008; Lippold, Fitzsimmons, Foote, Ratcliffe, \& Mennill, 2008). In natural settings, songs are thought to be produced mainly by males (Ficken, 1981), but recent evidence suggests that females also sing (Hahn et al., 2015; Hahn, Krysler, \& Sturdy, 2013). In the context of territorial defense, pairs of familiar neighbors commonly have been observed singing when they are both approaching a territory boundary, when they are both leaving a territory boundary, and when they are both remaining at fixed distances from each other at locations not near a territory boundary (Fitzsimmons et al., 2008; Lippold et al., 2008; Stefanski, 1967). Solo singing is also quite common (Mennill \& Otter, 2007); in some cases, this appears to serve to attract missing mates or young progeny (Dixon \& Stefanski, 1970; Odum, 1941). Generally, fee-bee songs appear to be used when birds are separated by at least $10 \mathrm{~m}$ (Dixon \& Stefanski, 1970). It is clear from these observations that fee-bee songs play an important role in mediating social interactions, particularly when interacting birds are not visible to one another. Although the song traditionally has been described as a "summons" (Odum, 1941), recent observations made with an acoustic array suggest that interacting chickadees also often sing when they are moving apart (Fitzsimmons et al., 2008). Regardless of what other functions the fee-bee song may serve, the evidence to date strongly indicates that the song provides a way for listening chickadees to monitor changes in the location and actions of the singer. It is thus surprising that some researchers have concluded, based on acoustic and playback experiments, that fee-bee songs are ill suited for this purpose (Fotheringham \& Ratcliffe, 1995).

Our findings suggest, instead, that many of the acoustic properties of the fee-bee song are conducive to providing information about the distance of the singer, if listeners compare the level of a received bee note to the level of coincident reverberation from the preceding fee note. Current methods are inadequate for objectively measuring what any individual chickadee knows about the movements of other birds. Laboratory experiments can reveal, however, whether chickadees are able to make the kinds 
of fine auditory distinctions in two-tone sounds that they would need to make in order to judge a singer's distance using coincident reverberation cues from fee-bee songs.

One subtle feature of the fee-bee song that has received relatively little attention to date is a brief amplitude dip in the approximate middle of the bee note, which can vary in duration within and across individuals (Fotheringham \& Ratcliffe, 1995; Hoeschele et al., 2010; Lohr, 2008). This feature has led some researchers to describe the song as the "fee-bee-ee" or "hey-sweetie" song (Kroodsma et al., 1999). Lohr (2008) suggested that this feature might serve as a ranging cue, since reverberation from the bee note produced prior to the dip would tend to fill in the gap as a singer's distance from a listener increased (i.e., larger dips correspond to shorter ranges). Chickadees appear to have some control over the presence and duration of this amplitude modulation, because it is not present in all bee notes (Kroodsma et al., 1999). If singers vary their bee breaks across song repetitions, then the duration or absence of this gap will be unreliable as a ranging cue unless listeners know what each song was like at the source. On the other hand, if listeners are using coincident reverberation cues to range singers, then singers can potentially use these gaps to transmit information beyond just their own position. In particular, temporarily decreasing the amplitude of the bee note will decrease the difference between reverberant and directly received acoustic energy, thereby providing two different cues correlated with source distance one corresponding to the actual singer's distance and the second corresponding to a "virtual" distance. Studies monitoring when and how singing chickadees vary the gap within their bee notes are needed to clarify the role of this feature.

\section{Limitations and Future Directions}

One limitation of the acoustical measurements collected in this preliminary study is that they do not fully characterize the range of propagation paths experienced by chickadees. Undoubtedly, the range of propagation effects experienced by a wild chickadee will be greater than might be measured from a fixed-height microphone within an open field. Open-field measurements provide a conservative estimate of available reverberant cues, because wooded environments generate more reverberation than open environments; reverberant tails comparable to the ones analyzed here have been observed in various chickadee habitats (e.g., Fotheringham \& Ratcliffe, 1995). Nevertheless, additional measurements from a wider range of source-receiver configurations are clearly needed to fully assess the viability of coincident reverberation as a distance cue.

A second limitation of our acoustic experiment is that recordings were made on a single day at night. It is known that vertical temperature profiles vary with time of day in ways that affect how sounds propagate, and that variations in atmospheric conditions can also affect propagation (Huisman \& Attenborough, 1991). It is unclear how such variations would affect the attenuation or reverberation of fee-bee songs in habitats frequented by chickadees, or the extent to which this might interfere with perception and assessment of any auditory distance cues within these songs. It is impractical to test all the different environmental conditions within which chickadees are likely to hear songs, so computer simulations of song propagation and reverberation likely will be needed to fully explore the effects of different conditions on the viability of different distance cues.

The fact that coincident reverberation cues can be found within chickadee fee-bee songs suggests that similar coincident reverberation cues may also be present within other animals' long-range vocalizations. For instance, Carolina chickadees (Kroodsma, Albano, Houlihan, \& Wells, 1995; Lohr, Nowicki, \& Weisman, 1991), and mountain chickadees (Lohr, 2008) produce songs with acoustic characteristics similar to those of the fee-bee song produced by black-capped chickadees. Animals that live in highly reverberant, low visibility environments and that often coordinate their movements (e.g., whales and dolphins) would be the most likely to benefit from the use of such cues. Consistent with this possibility, recent analyses of songs produced by humpback whales revealed that individual sounds within their songs also generate reverberant tails that overlap with subsequent sounds, and that ratios of frequencies across consecutive sounds are similar to those found in chickadee songs (Mercado, 2016). Closer examination of correlations between the spectra of sequential sounds produced by other species 
that acoustically communicate over long distances may further clarify the extent to which such auditory distance cues enable organisms to better coordinate their actions when visual inputs are degraded.

\section{Acknowledgments}

This work was supported in part by a student research grant to M. Wisniewski from NSF grant \#SMA-1041755 to the Temporal Dynamics of Learning Center, an NSF Science of Learning Center, and by a Natural Sciences and Engineering Research Council of Canada (NSERC) Discovery Grant and Discovery Accelerator Supplement, an Alberta Ingenuity Fund (AIF) New Faculty Grant, a Canada Foundation for Innovation (CFI) New Opportunities Fund (NOF) and Infrastructure Operating Fund (IOF) grants along with start-up funding and CFI partner funding from the University of Alberta (UofA) to CBS. LMG was supported by an Izaak Walton Killam Memorial Scholarship (IWKMS) at UofA and is currently a BBSRC Anniversary Future Leader Fellow. AHH is supported by a Michael Guyer Postdoctoral Fellowship from the Department of Zoology at the University of Wisconsin-Madison. All animal studies were conducted in accordance with the Canadian Council on Animal Care Guidelines and Policies with approval from the Animal Care and Use Committee for Biosciences for the University of Alberta and the Life and Environmental Sciences Animal Care Committee for the University of Calgary Life. Chickadees were captured and the research was carried out under an Environment Canada Canadian Wildlife Service Scientific permit, Alberta Fish and Wildlife Capture and Research permits, and City of Edmonton Parks Permit.

\section{References}

Avey, M. T., Quince, A. F., \& Sturdy, C. B. (2008). Seasonal and diurnal patterns of black-capped chickadee (Poecile atricapillus) vocal production. Behavioural Processes, 77, 149-155. doi:10.1016/j.beproc.2007.12.004

Bernstein, L. R., \& Green, D. M. (1988). Detection of changes in spectral shape: Uniform vs. non-uniform background spectra. Hearing Research, 34, 157-166.

Bregman, M. R., Patel, A. D., \& Gentner, T. Q. (2016). Songbirds use spectral shape, not pitch, for sound pattern recognition. Proceedings of the National Academy of Sciences USA, 113, 1666-1671.

Christie, P. J., Mennill, D. J., \& Ratcliffe, L. M. (2004a). Chickadee song structure is individually distinctive over long broadcast distances. Behaviour, 141, 101-124.

Christie, P. J., Mennill, D. J., \& Ratcliffe, L. M. (2004b). Pitch shifts and song structure indicate male quality in the dawn chorus of black-capped chickadees. Behavioral Ecology and Sociobiology, 55, 341-348.

Connor, R. C. (2000). Group living in whales and dolphins. In J. Mann, R. C. Connor, P. L. Tyack \& H. Whitehead (Eds.), Cetacean societies: Field studies of dolphins and whales (pp. 199-218). Chicago: University of Chicago Press.

Cynx, J., Williams, H., \& Nottebohm, F. (1990). Timbre discrimination in zebra finch (Taeniopygia guttata) song syllables. Journal of Comparative Psychology, 104, 303-308.

Dabelsteen, T., Larsen, O. N., \& Pedersen, S. B. (1993). Habitat-induced degradation of sound signals: Quantifying the effect of communication sounds and bird location on blur ratio, excess attenuation, and signal-to-noise ratio in blackbird song. Journal of the Acoustical Society of America, 93, 2206-2220.

Dai, H., \& Green, D. M. (1992). Auditory intensity perception: Successive versus simultaneous, across-channel discriminations. Journal of the Acoustical Society of America, 91, 2845-2854.

Dixon, K. L., \& Stefanski, R. A. (1970). An appraisal of the song of the black-capped chickadee. Wilson Bulletin, $82,53-62$.

Feth, L. L., \& O'Malley, H. (1977). Two-tone auditory spectral resolution. Journal of the Acoustical Society of America, 62, 940-947.

Ficken, M. S. (1981). What is the song of the black-capped chickadee? Condor, 83, 384-386.

Fitzsimmons, L. P., Foote, J. R., Ratcliffe, L. M., \& Mennill, D. J. (2008). Frequency matching, overlapping and movement behaviour in diurnal countersinging interactions of the black-capped chickadees. Animal Behaviour, 75, 1913-1920. 
Fotheringham, J. R., \& Ratcliffe, L. M. (1995). Song degradation and estimation of acoustic distance in blackcapped chickadees (Parus atricapillus). Canadian Journal of Zoology, 73, 858-868.

Gammon, D. E., \& Baker, M. C. (2004). Song repertoire evolution and acoustic divergence in a population of blackcapped chickadees. Animal Behaviour, 68, 903-913.

Gammon, D. E., Baker, M. C., \& Tipton, J. R. (2005). Cultural divergence within novel song in the black-capped chickadee (Poecile atricapillus). Auk, 122, 853- 871.

Green, D. M. (1987). Profile analysis: Auditory intensity discrimination. New York: Oxford University Press.

Hahn, A. H., Guillette, L. M., Hoeschele, M., Otter, K. A., Ratcliffe, L. M., \& Sturdy, C. B. (2017). Discrimination of male black-capped chickadee songs: Relationship between acoustic preference and performance accuracy. Animal Behaviour, 126, 107-121.

Hahn, A. H., Hoang, J., McMillan, N., Campbell, K., Congdon, J., \& Sturdy, C. B. (2015). Biological salience influences performance and acoustic mechanisms for the discrimination of male and female songs. Animal Behaviour, 104, 213-228.

Hahn, A. H., Hoeschele, M., Guillette, L. M., Hoang, J., McMillan, N., Congdon, J. V., ...Grava, T. (2016). Blackcapped chickadees categorize songs based on features that vary geographically. Animal Behaviour, 112, 93-104.

Hahn, A. H., Krysler, A., \& Sturdy, C. B. (2013). Female song in black-capped chickadees (Poecile atricapillus): Acoustic song features that contain individual identity information and sex differences. Behavioural Processes, 98, 98-105.

Henry, K. S., \& Lucas, J. R. (2010). Auditory sensitivity and the frequency selectivity of auditory filters in the Carolina chickadee, Poecile carolinensis. Animal Behaviour, 80, 497-507.

Hoeschele, M., Cook, R. G., Guillette, L. M., Hahn, A. H., \& Sturdy, C. B. (2014). Timbre influences chord discrimination in black-capped chickadees (Poecile atricapillus) but not humans (Homo sapiens). Journal of Comparative Psychology, 128, 387-401.

Hoeschele, M., Moscicki, M. K., Otter, K. A., van Oort, H., Fort, K. T., Farrell, T. M., ...Sturdy, C. B. (2010). Dominance signalled in an acoustic ornament. Animal Behaviour, 79, 657-644.

Holland, J., Dabelsteen, T., Pedersen, S. B., \& Paris, A. L. (2001). Potential ranging cues contained within the energetic pauses of transmitted wren song. Bioacoustics, 12, 3-20.

Huisman, W. H. T., \& Attenborough, K. (1991). Reverberation and attenuation in a pine forest. Journal of the Acoustical Society of America, 90, 2664-2677.

Kolarik, A. J., Moore, B. C., Zahorik, P., Cirstea, S., \& Pardhan, S. (2016). Auditory distance perception in humans: A review of cues, development, neuronal bases, and effects of sensory loss. Attention, Perception, \& Psychophysics, 78, 373-395. doi: 10.3758/s13414-015-1015-1

Kroodsma, D. E., Albano, D. J., Houlihan, P. W., \& Wells, J. A. (1995). Development by black-capped chickadee (Parus atricapillus) and Carolina chickadees (P. carolinensis). Auk, 112, 29-43.

Kroodsma, D. E., Byers, B. E., Halkin, S. L., Hill, C., Minis, D., Bolsinger, J. R., ..Wilda, K. (1999). Geographic variation in black-capped chickadee songs and singing behavior. Auk, 116, 387-402.

Langemann, U., Zokoll, M. A., \& Klump, G. M. (2005). Analysis of spectral shape in the barn owl auditory system. Journal of Comparative Physiology A: Neuroethology, Sensation, Neural, and Behavioral Physiology, 191, 889-901.

Larsen, E., Iyer, N., Lansing, C. R., \& Feng, A. S. (2008). On the minimum audible difference in direct-toreverberant energy ratio. Journal of the Acoustical Society of America, 124, 450-461.

Lippold, S., Fitzsimmons, L. P., Foote, J. R., Ratcliffe, L. M., \& Mennill, D. J. (2008). Post-contest behaviour in black-capped chickadees (Poecile atricapillus): Loser displays, not victory displays, follow asymmetrical countersinging exchanges. Acta Ethologica, 11, 67-72.

Lohr, B. (2008). Pitch-related cues in the songs of sympatric mountain and black-capped chickadees. Behavioural Processes, 77, 156-165.

Lohr, B., \& Dooling, R. J. (1998). Detection of changes in timbre and harmonicity in complex sounds by zebra finches (Taeniopygia guttata) and budgerigars (Melopsittacus undulatus). Journal of Comparative Psychology, 112, 36-47.

Lohr, B., Nowicki, S., \& Weisman, R. (1991). Pitch production in Carolina chickadee songs. Condor, 93, 197-199.

Mennill, D. J., \& Otter, K. A. (2007). Status signalling and communication networks in chickadees: Complex communication with a simple song. In K. A. Otter (Ed.), The ecology and behavior of chickadees and titmice: An integrated approach (pp. 215 - 233): Oxford: Oxford University Press.

Mercado, E., III. (2016). Acoustic signaling by singing humpback whales (Megaptera novaeangliae): What role does reverberation play? PLoS One, 11, e0167277. doi: 10.1371/journal.pone.0167277 
Morton, E. S. (1982). Grading, discreteness, redundancy, and motivation-structural rules. In D. E. Kroodsma \& E. H. Miller (Eds.), Acoustic communication in birds (pp. 183 - 212). New York: Academic Press.

Morton, E. S. (1986). Predictions from the ranging hypothesis for the evolution of long distance signals in birds. Behaviour, 99, 65-86.

Morton, E. S. (2012). Putting distance back into bird song with mirror neurons. Auk, 129, 560-564.

Naguib, M. (2003). Reverberation of rapid and slow trills: Implications for signal adaptations to long-range communication. Journal of the Acoustical Society of America, 113, 1749-1756.

Naguib, M., \& Wiley, R. H. (2001). Estimating the distance to a source of sound: Mechanisms and adaptations for long-range communication. Animal Behaviour, 62, 825-837.

Nelson, B. S. (2000). Avian dependence on sound pressure level as an auditory distance cue. Animal Behaviour, 59, 57-67.

Nelson, B. S., \& Stoddard, P. K. (1998). Accuracy of auditory distance and azimuth perception by a passerine bird in a natural habitat. Animal Behaviour, 56, 467-477.

Nowicki, S. (1983). Flock-specific recognition of chickadee calls. Behavioral Ecology and Sociobiology, 12, 317320.

Odum, E. P. (1941). Annual cycle of the black-capped chickadee. Auk, 58, 314-333.

Padgham, M. (2004). Reverberation and frequency attenuation in forests--implications for acoustic communication in animals. Journal of the Acoustical Society of America, 115, 402-410.

Phillmore, L. S., Sturdy, C. B., Turyk, M. R., \& Weisman, R. (2002). Discrimination of individual vocalizations by black-capped chickadees (Poecile atricapilla). Animal Learning \& Behavior, 30, 43-52.

Phillmore, L. S., Sturdy, C. B., \& Weisman, R. G. (1998). Discrimination of auditory distance cues by black-capped chickadees (Poecile atricapillus) and zebra finches (Taeniopygia guttata). Journal of Comparative Psychology, 112, 282-291.

Pohl, N. U., Klump, G. M., \& Langemann, U. (2015). Effects of signal features and background noise on distance cue discrimination by a songbird. Journal of Experimental Biology, 218, 1006-1015. doi: 10.1242/jeb.113639

Radziwon, K. E., Welch, T. E., Cone, J. P., \& Dent, M. L. (2011). Identification of auditory distance cues by zebra finches (Taeniopygia guttata) and budgerigars (Melopsittacus undulatus). Journal of the Acoustical Society of America, 129, 3384-3392.

Richards, D. G., \& Wiley, R. H. (1980). Reverberations and amplitude fluctuations in the propagation of sound in a forest: Implications for animal communication. American Naturalist, 115, 381-399.

Shackleton, S. A., Ratcliffe, L. M., \& Weary, D. M. (1992). Relative frequency parameters and song recognition in black-capped chickadees. Condor, 94, 782-785.

Slabbekoorn, H., Ellers, J., \& Smith, T. B. (2002). Birdsong and sound transmission: The benefits of reverberations. Condor, 104, 564-573.

Smith, S. M. (1991). The black-capped chickadee: Behavioral ecology and natural history. Cornell, NY: Cornell University Press.

Stefanski, R. A. (1967). Utilization of the breeding territory in the black-capped chickadee. Condor, 69, 259-267.

Versfeld, N. J., \& Houtsma, A. J. (1995). Discrimination of changes in the spectral shape of two-tone complexes. Journal of the Acoustical Society of America, 98, 807-816.

Weisman, R., \& Ratcliffe, L. (1989). Absolute and relative pitch processing in black-capped chickadees (Parus atricapillus). Animal Behaviour, 38, 685-692.

Weisman, R., \& Ratcliffe, L. (2004). Relative pitch and the song of the black-capped chickadees. American Scientist, 92, 532-539.

Weisman, R., Ratcliffe, L., Johnsrude, I., \& Hurly, T. A. (1990). Absolute and relative pitch production in the song of the black-capped chickadee. Condor, 92, 118-124.

Wiley, R. H. (1998). Ranging reconsidered. Behavioral Ecology and Sociobiology, 42, 143-146.

Wiley, R. H., \& Richards, D. G. (1978). Physical constraints on acoustic communication in the atmosphere: Implications for the evolution of animal vocalizations. Behavioral Ecology and Sociobiology, 3, 69-94.

Wiley, R. H., \& Richards, D. G. (1982). Adaptations for acoustic communication in birds: Sound transmission and signal detection. In D. E. Kroodsma \& E. H. Miller (Eds.), Acoustic communication in birds (pp. 132-163). New York: Academic Press.

Wong, A., \& Gall, M. D. (2015). Frequency sensitivity in the auditory periphery of male and female black-capped chickadees (Poecile atricapillus). Zoology, 118, 357-363. 
Yang, H. S., Kang, J., Cheal, C., Van Renterghem, T., \& Botteldooren, D. (2013). Quantifying scattered sound energy from a single tree by means of reverberation time. Journal of the Acoustical Society of America, 134, 264-274.

Zahorik, P. (2002). Direct-to-reverberant energy ratio sensitivity. Journal of the Acoustical Society of America, 112, 2110-2117. 